\title{
Pour une Suisse sans rougeole
}

Interview avec la Dr Nicole Pellaud, médecin en pédiatrie, $\mathrm{FMH}$, et présidente de la Société suisse de pédiatrie. Dr Pellaud est membre du Comité national pour une Suisse sans rougeole, qui a été convoqué en 2013 dans le cadre de la stratégie nationale d'élimination de la rougeole. Le comité sert de plateforme de soutien pour la mise en œuvre de la stratégie, s'occupe de tâches de relations publiques et certifie l'élimination de la rougeole de la Suisse selon les critères de l'OMS.

Sabine Walser

Co-cheffe de la section Epidémiologie,

Office fédéral de la santé publique, responsable de la surveillance de la rougeole, des oreillons et de la rubéole
Correspondance:

Dr sc. nat. Sabine Walser Office fédéral de la santé publique Schwarztorstrasse 96 CH-3003 Berne Tél. 0313250534 Fax 0313238795

\begin{abstract}
Madame Pellaud, vous vous engagez pour le comité pour une Suisse sans rougeole. Quelles en sont les motivations?

Nous avons suffisamment de données probantes nous indiquant que la rougeole est dangereuse et qu'il est possible d'en protéger la population par la vaccination. En tant que médecins, nous avons le devoir de nous engager autant pour la santé individuelle que publique.
\end{abstract}

Comment voyez-vous votre contribution personnelle pour ce comité?

En soutenant dans mon milieu professionnel et auprès du grand public la promotion des mesures pertinentes définies par nos experts, telles que la vaccination, l'identification rapide des cas et les mesures en cas de flambées, et en transmettant au comité des pistes d'action issues de mon expérience professionnelle.

Comment la Suisse peut-elle parvenir à l'élimination de la rougeole d'ici à 2015 ?

Toutes les parties doivent se sentir concernées et s'y engager - tous les cantons, tous les professionnels de la santé, et surtout la population. Il y a là différents enjeux pour chacune des parties: responsabilité, communication, confiance.

Vous êtes pédiatre de profession. Comment les pédiatres devraient-ils contribuer à l'élimination de la rougeole?

Les pédiatres le font déjà activement et la plupart d'entre eux appliquent le plan suisse de vaccination avec deux doses de ROR à 12 et 15 mois, en informant les parents, en leur recommandant les vaccinations et en les réalisant.

Comment procédez-vous dans une consultation, lorsque les parents ne sont pas sûrs de vouloir faire vacciner leur enfant? Cela se joue surtout dans la relation de confiance; si les parents se sentent respectés dans leurs interrogations, nous avons l'opportunité d'offrir une information leur donnant l'occasion de réfléchir et de les orienter au mieux dans leur choix.

Les chiffres de la couverture vaccinale suisses démontrent que la deuxième dose de vaccin est encore souvent oubliée. Comment garantissez-vous que tou(te)s vos patient(e)s obtiennent les deux doses de vaccination?

En proposant un rendez-vous vaccinal pour la $2^{\mathrm{e}}$ dose à l'issue de la $1^{\text {ère, }}$ en rappelant aux parents de reprendre rendez-vous si nécessaire, et en ayant en tête la vérification du statut vaccinal lors des consultations.
Il y a déjà eu deux flambées de rougeole relativement importantes cette année. Quelles mesures préventives prendriezvous dans votre cabinet, si vous diagnostiquiez la rougeole lors de vos heures de consultation?

Isolement immédiat de l'enfant atteint, identification des contacts au cabinet et en famille avec rattrapage vaccinal si nécessaire; identification des enfants de moins de six mois parmi les contacts pour immunoglobulines si indiqué; liste des lieux fréquentés avec contacts proches; déclaration au médecin cantonal, annonce du cas au médecin scolaire le cas échéant.

Les milieux contre les vaccins critiquent le fait que l'industrie pharmaceutique soit derrière l'élimination de la rougeole. Quel est votre avis là-dessus?

La rougeole décime des populations d'enfants dans les pays en voie de développement, pour lesquels l'élimination de la rougeole est une priorité de longue date. La vaccination est nécessaire pour protéger ces enfants comme les nôtres et n'est nullement une manigance imaginée par l'industrie, dans le but de faire des affaires. Pour ce qui concerne la Suisse, la Société Suisse de Pédiatrie défend les intérêts touchant à la santé des enfants et des adolescents et suit les directives de l'Académie suisse des sciences médicales intégrées dans le code de déontologie $\mathrm{FMH}$, notamment celles liées à la collaboration avec l'industrie pharmaceutique. Nos membres s'inscrivent dans cette démarche. Parmi eux, nous comptons des pédiatres experts en infectiologie qui participent à l'élaboration de recommandations basées sur l'évidence, tel que c'est le cas pour la rougeole. Nous plaçons notre confiance dans leur respect des directives précitées, et de surcroît, étant dans un petit pays où tout le monde se connaît, nous savons qu'ils la méritent.

Comment réagissez-vous face aux parents, qui déclarent vouloir renoncer au vaccin, laisser leurs enfants attraper la rougeole, pour être par la suite protégés plus longtemps?

Ici aussi, il s'agit d'une question liée à la relation de confiance. Mais s'ils ont des convictions, il est important de comprendre les soucis légitimes de parents qui veulent le bien de leur enfant, de fournir des informations précises et répondant à leurs préoccupations, de leur transmettre ce que nous savons sans être des ayatollahs... 